Proceedings

\title{
Reading Urban Transformation Based on Geolocated Crowdsourced Data
}

\author{
Álvaro Bernabeu-Bautista ${ }^{1,}{ }^{*}$, Mariana Huskinson ${ }^{1}$ and Leticia Serrano-Estrada ${ }^{1}$ \\ 1 Building Sciences and Urbanism Department, University of Alicante; alvaro.bautista@ua.es (Á.B.-B.), \\ mh65@gcloud.ua.es (M.H.), leticia.serrano@ua.es (L.S.-E.) \\ * Correspondence: alvaro.bautista@ua.es
}

Citation: Bernabeu-Bautista, A.; Huskinson, M.; Serrano-Estrada, L. 2022, Reading Urban Transformation Based on Geolocated Crowdsourced Data. SUPTM 2022 conference proceedings sciforum- 054436 .

https://doi.org/10.31428/10317/10581

Publisher's Note: UPCT and Sciforum stays neutral with regard to jurisdictional claims in published maps and institutional affiliations.

Copyright: (c) 2022 by the authors. Submitted for possible open access publication under the terms and conditions of the Creative Commons Attribution (CC BY) license (https://creativecommons.org/license s/by/4.0/)
Abstract: Social and economic instability in cities caused by the pandemic emergency has highlighted the necessity of carrying out diagnoses to identify courses of action for the purpose of reducing the vulnerability of urban areas in the face of future crisis. This research aims to identify how the impact of the COVID-19 pandemic and the measures adopted have influenced urban and economic activities and the use, presence and preferences of urban public spaces. Specifically, an analysis in several cities before and after the most critical phases of the pandemic is proposed based on virtual social network geodata. Since each individual represents a node of information exchange, it is hypothesized that a comparison between the data generated before and after the pandemic offer a first glance at how changes in the social (collective behaviors and preferences) and physical (economic and urban activities) dimensions in urban environments have been produced. The results provide a descriptive and contrasted analysis that evidences the relevance of using crowdsourced data for monitoring the changes and transformations that took place as a result of the pandemic crisis in order to inform decision-making for future urban policies and actions.

Keywords: COVID-19; crowdsourced data; urban transformation, monitoring

\section{Introduction}

This research is temporally related to the global wave of the COVID-19 pandemic, first initiated in Wuhan, China [1] in November 2019, which has radically impacted and transformed the socio-spatial structure of cities. The consequences of the measures adopted by worldwide governments have promoted a new spatiotemporal reality in practically all scales of the urban dimension [2]. For instance, the mobility restrictions and the social distance have had an impact on the use, activities and interpersonal relationships that occur in the urban public space.

Social media data have proven to be very useful to obtain an updated view on collective behaviors and perceptions when tackling situations of crisis [3,4], a fact that, combined with the increasing amount and availability of information shared in recent months as a clear consequence of the COVID-19 pandemic [5], has attracted scientific interest in measuring the impact and consequences that the epidemic has left on social, spatial and urban realities. Some research carried out so far in this line of work has addressed the trends in citizen movement patterns during different timeframes in the critical phases of the pandemic from Twitter data in diverse geographical contexts [6,7]; the spatiotemporal distribution of COVID-19 cases in Wuhan, China using data from Sina Weibo social network [1]; the online social activity and the most recurrent themes during the pandemic in Poland [4]; the identification of collective feelings and opinions towards the different governmental measures adopted by India and Spain to face the crisis from Twitter texts [8] or, the variations in the rhythms of human activity as a consequence of the extreme confinement in France through YouTube videos and Twitter data [9]. 
However, there is still a long way to go to fully understand the changes that the pandemic has brought into the city dynamics. This information is key to guide present and future interventions for preparing the urban setting, making it more resilient to future challenges. In this line of research, this study seeks to contribute to the existing knowledge on the extent to which the pandemic has had an impact on urban and economic activities as well as on the use and collective perception of public spaces at a neighborhood scale. Specifically, the objective is to get a first insight of the extent to which the city has been transformed regarding economic and urban activities, citizen preferences and people presence in urban spaces by comparing before and after COVID-19 scenarios as reflected in geolocated social media sourced data.

\section{Case Study and Sources}

\subsection{Area of study}

Two neighborhoods from four different cities are select as study cases. On the one hand, the intent is to make a comparison between local and international contexts that have reacted politically, socially and, therefore, spatially differently to the COVID-19 crisis and, on the other hand, the neighborhoods selected within each city have very different socioeconomic conditions. The cities and neighborhoods selected are shown in Table 1.

Table 1. Cities and neighborhoods selected as case studies

\begin{tabular}{ccc}
\hline Country & City & Neighborhoods \\
\hline \multirow{2}{*}{ Spain } & Valencia & Ensanche \\
& & Poblats Marítims \\
& Centro \\
& Alicante & Juan XXIII \\
\hline \multirow{2}{*}{ Mexico } & Mexico City & Condesa \\
& Gztapalapa \\
\hline \multirow{2}{*}{ Sweden } & Gothenburg & Skår \\
& & Hjällbo/Hammarkullen \\
\hline
\end{tabular}

While Spain government adopted strict measures during the lockdown, Sweden has adopted more relaxed measures, although it was the country with the most recorded deaths in the Scandinavian region. Mexico has very different conditions from the other case studies and a critical situation regarding the COVID-19 average contagion rate, but with the enormous difficulty of adopting extreme measures to close the activity due to the country's economic fragility.

\subsection{Data sources}

Various social media data sources have been used for this study. With respect to the diversity of social networks currently available, two conditions were relevant when selecting the potential data sources: (i) first, they had to provide information about where and when human activity occurs and (ii) second, they offer information about the characteristics of the places. In the authors' experience, several social networks that meet these two conditions have been identified [10]. These sources offer a broad range of information that allows, on the one hand, to approach urban phenomena from diverse perspectives and, on the other hand, to overcome some limitations related, for instance, to the representativeness of some social networks in certain areas [11].

Specifically, this research is focused on three virtual social networks: Google Places, Foursquare and Twitter, and the data from these three sources are considered as indicators of urban and economic activity, citizen perception and preferences, and citizen opinions and presence, respectively. 


\section{Method}

The method mainly comprised the following steps: (i) data collection before and after the pandemic; (ii) data validation; (iii) data visualization; and (iv) data comparison and first insights.

The Foursquare, Twitter and Google Places datasets before and after the pandemic were collected through their API (application programming interface) using a web-based application designed for that purpose: SMUA (Social Media Urban Analyzer) [12]. After data were retrieved, a validation and verification process was needed to eliminate duplicated registers and non-human generated content (like tweets from bots that are generated automatically).

Data was visualized following two strategies: quantifying de number of data using a $25 \mathrm{~m} \times 25 \mathrm{~m}$ grid and comparing the difference of data density on each cell from the grid before and after pandemic. The reference grid was used to appreciate with more granularity the changes in the different areas analyzed. It is considered that $25 \mathrm{~m}$ is a threshold distance that is frequently used in the discipline to measure issues related to the quality of the urban environment, for example, with aspects related to urban accessibility [13].

Finally, the results from the before and after COVID-19 scenarios were compared and initial insights regarding the variation of urban activity before and after the pandemic were drawn.

\section{Results}

With regard to the changes in the economic activities of the case studies analyzed, Google Places data showed an important difference between the amount of postcovid and precovid data. Indeed, a significant loss of activity in a generalized manner throughout the selected neighborhoods was evidenced, specially near the main commercial axes where a greater difference on the quantity of urban and economic activity can be appreciated.

With Foursquare data it was possible to quantify the cumulative number of users that have visited the venues of the neighbourhood and then make a comparison of these values before and after the pandemic. Indeed, it was observed that the increase in the number of users from the precovid to the postcovid data is quite significant in those venues related to relevant public spaces.

Twitter data provided insights on the presence of citizens in urban public spaces. The loss of tweets, and therefore, citizens' presence is appreciable, especially in urban axis that are usually active. These differences are significant in Mexico and Spain mainly due to the closure of establishments during the lockdown and the subsequent nighttime mobility restrictions.

It is also noteworthy that the number of generated data in all study cases is related to the socioeconomic level of each neighborhood as the low-income areas aggregates less registers than high-income areas, although the loss of urban activity and polarization phenomena is still appreciable in both cases.

\section{Conclusions}

This study offers some initial insights on the pre, and post COVID-19 changes related to urban and economic activities in two neighbourhoods of four different geographic contexts (Alicante and Valencia in Spain, Gothenburg in Sweden, and Mexico City in Mexico). Findings reveal that when comparing the density of activity of before and after COVID19 scenarios, no significant changes were observed, however when analysing the quantitative difference between the two, a significant loss of urban activity, including economic activity and citizens' presence, is notable. Moreover, through Foursquare data it was observed that the presence and preferences for certain economic and urban activities has 
become more polarized in areas where there was already some activity prior the pandemic breakout. Moreover, and as expected, public spaces had become more relevant since the pandemic while a decrease in urban vibrance has been observed in urban axis with predominantly commercial and services related activities. As far as the use of a reference grid for visualizing the differences, it has been an essential methodological step to pinpoint specific areas where activity has increased or decreased. These initial observations could lead to a future research that further analyses the microdata for a deeper understanding of which kind of urban and economic activities have been mostly affected by the pandemic crisis.

Funding: This research was co-funded by (i) Conselleria de Innovación, Universidades, Ciencia y Sociedad Digital, Generalitat Valenciana and European Social Fund (ACIF/2020/173) and (ii) Conselleria de Innovación, Universidades, Ciencia y Sociedad Digital, Generalitat Valenciana (GV/2021/177).

Conflicts of Interest: The authors declare no conflict of interest. 